Article

\title{
Intradialytic Cardiovascular Exercise Training Alters Redox Status, Reduces Inflammation and Improves Physical Performance in Patients with Chronic Kidney Disease
}

\author{
Apostolos Sovatzidis ${ }^{1, *(D)}$, Athanasios Chatzinikolaou ${ }^{2}$, Ioannis G. Fatouros ${ }^{3}$ (D), \\ Stylianos Panagoutsos ${ }^{1}$, Dimitrios Draganidis ${ }^{3}$ D, Eirini Nikolaidou ${ }^{4}$, Alexandra Avloniti ${ }^{2}$, \\ Yiannis Michailidis ${ }^{2}$, , Ioannis Mantzouridis ${ }^{2}$, Alexios Batrakoulis ${ }^{3} \mathbb{D}$, Ploumis Pasadakis ${ }^{1, *}$ \\ and Vassilis Vargemezis ${ }^{\dagger}$ \\ 1 Department of Nephrology, Faculty of Medicine, University Hospital of Alexandroupolis, \\ Democritus University of Thrace, 68100 Alexandroupolis, Greece; spanagou@med.duth.gr \\ 2 School of Physical Education and Sport Sciences, Democritus University of Thrace, 69100 Komotini, Greece; \\ athchatz.tefaa@gmail.com (A.C.); alavloni@phyed.duth.gr (A.A.); michailidis79@hotmail.com (Y.M.); \\ ioanmatz3@med.duth.gr (I.M.) \\ 3 School of Physical Education and Sport Sciences, University of Thessaly, Karies, 42100 Trikala, Greece; \\ fatouros@otenet.gr (I.G.F.); dimidraganidis@gmail.com (D.D.); alexis_batrakoulis_75@hotmail.com (A.B.) \\ 4 Department of Plastic, Reconstructive and Hand Surgery \& Burns ICU, General Hospital of Thessaloniki \\ “G. Papanikolaou”, 57010 Thessaloniki, Greece; eirininikolaidou7@gmail.com \\ * Correspondence: t.sovatzidis@gmail.com (A.S.); ploumis@med.duth.gr (P.P.); \\ Tel.: +30-693-6143-424 (A.S.); +30-255-1353-145 (P.P.); Fax: +30-255-1353-146 (A.S. \& P.P.) \\ $+\quad$ Passed away on October 2016.
}

Received: 10 August 2020; Accepted: 11 September 2020; Published: 15 September 2020

check for updates

\begin{abstract}
Redox status (RS) perturbations and inflammation are fundamental features of chronic kidney disease (CKD) that are substantially exacerbated in end-stage renal disease (ESRD). This study aimed at investigating the efficacy of a 6-month intradialytic exercise training program on RS, inflammation and physical performance in patients with ESRD. Twenty hemodialysis (HD) patients (17 males, three females) were randomly assigned to either an intradialytic training (bedside cycling) group (TR; $n=10)$ or a control group (CON; $n=10)$ for 6 months. Anthropometrics [body mass and height, body mass index (BMI), body composition], physical performance $\left(\mathrm{VO}_{2 \text { peak }}\right)$, functional capacity [North Staffordshire Royal Infirmary (NSRI) walk test, sit-to-stand test (STS-60)], quality of life (short form-36 (SF-36) as well as RS [thiobarbituric acid reactive substances (TBARS), protein carbonyls (PC), reduced (GSH) and oxidized (GSSG) glutathione, GSH/GSSG, total antioxidant capacity (TAC), catalase activity (CAT)] and high-sensitivity C-reactive protein (hs-CRP) were assessed at baseline and after the 6-month intervention. Peak oxygen consumption $\left(\mathrm{VO}_{2 \text { peak }}\right)$ increased by $15 \%$ only in TR $(p<0.01)$. Performance in NSRI, STS-60 and SF-36 improved by $4-13 \%$ only in TR $(p<0.01)$. Exercise training reduced TBARS (by $28 \%$ ), PC (by 31\%) and hs-CRP (by 15\%), and elevated GSH (by 52\%), GSH/GSSG (by 51\%), TAC (by 59\%) and CAT (by 15\%) ( $p<0.01$ ). These findings suggest that engagement in chronic intradialytic cardiovascular exercise alters RS, reduces inflammation and improves performance in patients with ESRD.
\end{abstract}

Keywords: chronic kidney disease; hemodialysis; exercise; training; oxidative stress; inflammation 


\section{Introduction}

Kidney diseases are recognized as a tremendous public health burden, with their prevalence exceeding 850 million patients globally [1]. Of note, (CKD), the most common form of kidney disease, affects $8-16 \%$ of adult population [2] and is expected to be the 5th leading cause of death worldwide by 2040 [3]. CKD is characterized by lasting damage to kidneys or decreased glomerular filtration rate (GFR) [2] for more than three months [4] and is classified into five stages according to the estimated GFR. According to the United States Renal Data System, the fifth stage, also known as end-stage renal disease (ESRD), led almost $87 \%$ of these patients to the most widely used Renal Replacement Therapy (RRT) (i.e., hemodialysis) in 2017 [5].

Oxidative stress (OS) and (RS) perturbations are distinctive features of CKD that are evident even in the early stage of the disease [6,7] and increase progressively as the disease progresses [8]. Patients with ESRD, a high percentage of whom are older adults, display increased pro-oxidant activity, which is largely attributed to coexisting comorbidities such as diabetes mellitus (DM), hypertension, metabolic syndrome and atherosclerosis $[9,10]$. On the other hand, the anti-oxidant capacity of these patients is impaired since reduced levels of vitamin C, D, E, and selenium are received due to (i) dietary restriction of fruits and vegetables (in order to avoid hyperkalemia), (ii) malnutrition and (iii) diminished intestinal absorption, while a deficiency of the redused glutathione (GSH) scavenging system is also evident [9,11,12]. Furthermore, the perturbation of RS in favor of the pro-oxidant state, is further exacerbated by (HD) [9], as the HD process per se contributes to loss of antioxidant molecules and up to a 14-fold increase in the production of reactive oxygen species ROS, following each session $[11,13]$. Factors that affect the excessive HD-derived (ROS) production include dialyzer membranes, the type of vascular access, the duration of (HD)session, dialysate, anticoagulative and administered drugs [10]. In addition, it has been proposed that HD increases the production of hydrogen peroxide through stimulation of neutrophil burst [14]. Indeed, the development of chronic inflammation is also a hallmark of ESRD, as pro-oxidant molecules and pro-inflammatory mediators interact continuously in kidney diseases, propagating a vicious cycle that results in OS and inflammation [6,15].

Both ROS and inflammation are considered major contributors to endothelial dysfunction, thereby increasing the risk for atherosclerosis and cardiovascular-related morbidity and mortality in these patients [16-19]. Thus, the establishment of therapeutic interventions that could be efficiently utilized for the reduction of OS and chronic inflammation would be fundamental for the progression of CKD, particularly in patients undergoing HD. Exercise has been shown to drastically alter redox status and intracellular redox-dependent signaling pathways in various populations both acutely and following its systematic implementation [20-23]. In light of the beneficial effect of exercise training on OS and inflammatory status both in healthy [24,25] and other clinical populations [25], several studies investigated the efficacy of light-to-moderate-intensity intradialytic exercise training in improving the physical status and overall quality of life of the patients, reporting a favorable effect [26-31]. In addition, a few investigations have provided indications that engagement to long-term ( 3 to 4 months) exercise training reduces OS markers in patients with CKD [32-34], although acute exercise has been shown to exert a negative effect $[30,35]$.

Therefore, this study aimed at investigating changes in redox status and inflammatory indicators as well as physical performance and overall quality of life in patients with ESRD, following a 6-month intradialytic exercise training intervention.

\section{Materials and Methods}

\subsection{Participants and Experimental Design}

The present study aimed at investigating the efficacy of a 6-month intradialytic cardiovascular training intervention in altering RSand inflammation and improving physical performance in patients with ESRD. All (HD) patients from an outpatient HD unit were informed about the aim and procedures 
of study, but 28 of them volunteered to participate and were assessed for eligibility. To be included in the study, prospective participants had to meet the following inclusion criteria: (a) participation in chronic therapy for $\geq 12$ months prior to the study, (b) underwent 4-h HD sessions 3 times/week with standard bicarbonate dialysis using biocompatible membranes (low flux polysulfone), (c) hemoglobin levels of $\geq 11 \mathrm{~g} / \mathrm{dL}$ (Erythropoiesis-Stimulating Agents were administered to all patients), (d) no use of antioxidant supplements (i.e., vitamin E, statins or any other medication with antioxidant properties), (e) adequate nourishment (total serum protein $6.8 \pm 0.5 \mathrm{~g} / \mathrm{dL}$ and serum albumin $4.3 \pm 0.2 \mathrm{~g} / \mathrm{dL}$ ), (f) no residual renal function, and (g) ability to execute a stationary bike workout. Exclusion criteria included: (a) the presence of an active infectious/inflammatory disease, (b) uncontrolled hypertension and DM, (c) diseases that might interfere with exercise capacity or/and be exacerbated by activity such as ischemic cardiopathy or symptoms related to coronary artery disease, anemia (hemoglobin levels $<11 \mathrm{~g} / \mathrm{dL}$, Hct $<33 \%$ ), chronic lung disease and orthopedic disorders, (d) use of steroids, immunosuppressives, and psychotropic agents, and (e) hospitalization within three months prior to the study. Accordingly, of the 28 patients who were initially recruited, 24 were included in the study and 20 finally completed it ( 1 transplantation, 2 dropouts, 1 death). The CONSORT flow diagram is presented in Figure 1 and participants' baseline characteristics are demonstrated in Table 1. Participants were informed about the aim of the study, the associated risks and benefits, and provided written informed consent. Procedures were in accordance with the 1975 Declaration of Helsinki and approved by the institutional review board for human research (22/02-06-2010).

\section{T. C O N S O R T}

CONSORT 2010 Flow Diagram

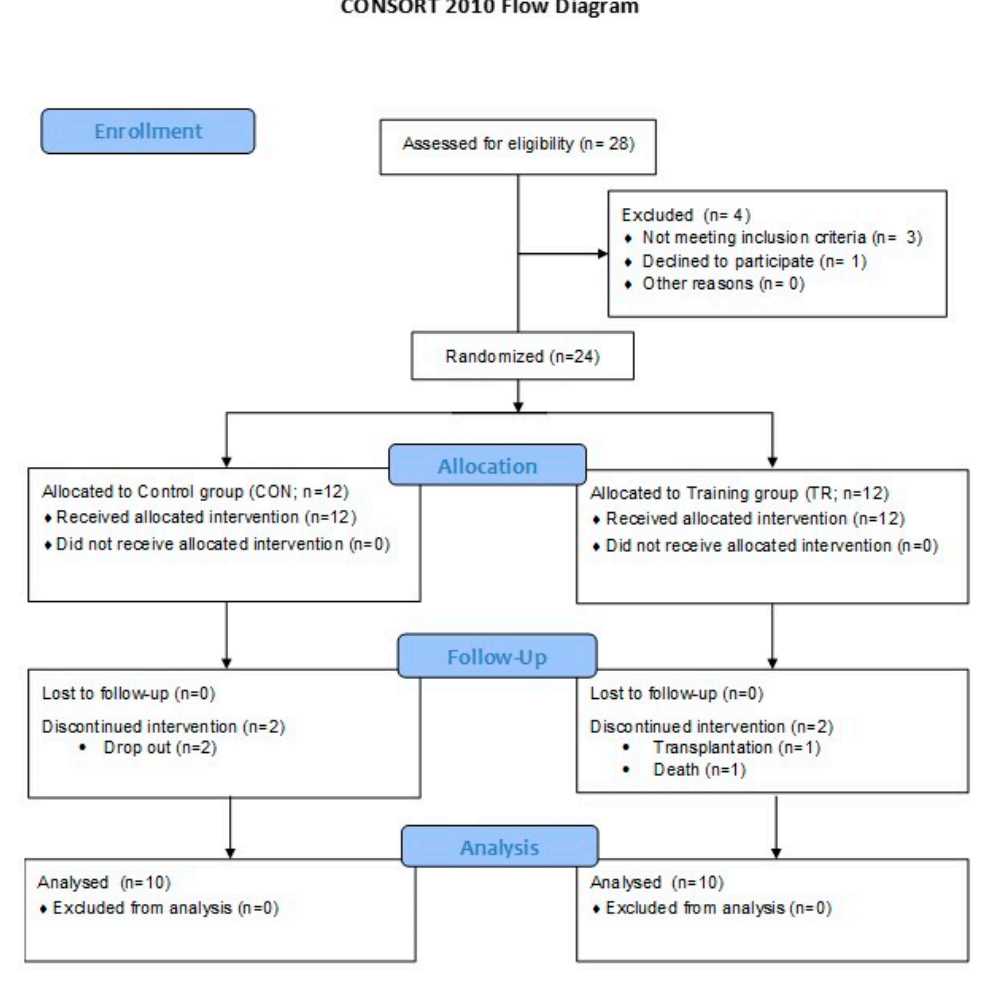

Figure 1. CONSORT flow diagram of the study.

A controlled, two-group, repeated measures design was used. Participants were randomly assigned to either a control group (CON, $N=10$, abstained from exercise training during HD sessions) or a training group (TR, $N=10$ ) that participated in a 6-month, supervised, intradialytic training intervention. Before and after the 6-month intervention period, all participants underwent assessment 
of their anthropometric profile, physical performance level, functional capacity, clinical status and provided a resting blood sample for the determination of OS, inflammatory profile, and antioxidant status. During intervention, CON received only the typical HD sessions, whereas the TR group participated in HD sessions plus in an intradialytic cardiovascular exercise training program.

Table 1. Participants' physical and clinical characteristics at baseline.

\begin{tabular}{ccc}
\hline Variables & Exercise Group $(\boldsymbol{n = 1 0 )}$ & Control Group $(\boldsymbol{n}=\mathbf{1 0})$ \\
\hline Gender (Female/Male) & $2 / 8$ & $1 / 9$ \\
Age (yr) & $52.8 \pm 17.1$ & $53 \pm 7,6$ \\
Body Height $(\mathrm{m})$ & $1.71 \pm 0.09$ & $1.71 \pm 0.1$ \\
Body Mass $(\mathrm{kg})$ & $72.5 \pm 14.6$ & $74.6 \pm 9.3$ \\
BMI $\left(\mathrm{kg} / \mathrm{m}^{2}\right)$ & $24.6 \pm 3.54$ & $25.5 \pm 1.84$ \\
Body Fat $(\%)$ & $27 \pm 2.27$ & $27.3 \pm 3.54$ \\
Dialysis History (months) & $88.8 \pm 9.9$ & $89.7 \pm 10.1$ \\
Residual Urea Clearance $\left(\mathrm{ml} / \mathrm{min}^{-1}\right)$ & $1.32 \pm 0,2$ & $1.28 \pm 0.3$ \\
Intradialytic Weight Gain $(\mathrm{kg})$ & $2.66 \pm 0.6$ & $2.55 \pm 0.6$ \\
Dialyzer Clearance of Urea $\left(\mathrm{K}_{\mathrm{t}} / \mathrm{V}\right)$ & $1.33 \pm 0.4$ & $1.27 \pm 0.3$ \\
\hline
\end{tabular}

BMI: Body Mass Index.

\subsection{Exercise Training Program}

HD patients in TR participated three times weekly in an intradialytic cardiovascular exercise program performed on a bedside cycle ergometer (Monark Rehab Trainer 881E, Varberg, Sweden) throughout the 6-month intervention period. Each exercise session started $60 \mathrm{~min}$ after the initiation of the HD session and consisted of 5 min warm-up, cycling at the desired workload for a self-selected time (depending on each participant's tolerance) and 5 min cool-down. The exercise intensity was individually adjusted based on the Borg rating scale (6-20 scale) of perceived exertion (RPE) and corresponding at an RPE of 11 ("light") to 13 ("somewhat hard"). Total workload was progressively increased during the 6-month intervention period, at a self-selected rate (i.e., every month participants were asked to increase the external resistance and the duration of exercise training based on their tolerance). Participants were continuously supervised and monitored by an exercise physiologist and a nephrologist, during each exercise session. Blood pressure, heart rate (HR), RPE and oxygen saturation $\left(\mathrm{SpO}_{2}\right)$ were monitored before, during and after the exercise protocol. Participants were allowed to participate in the exercise program only if they displayed: (i) controlled systolic blood pressure (SBP) and diastolic blood pressure (DBP) and (ii) resting $\mathrm{SpO}_{2} \geq 90 \%$. Indications and symptoms for the discontinuation of the exercise regime included: (i) chest pain, (ii) arrhythmias, (iii) dyspnea, (iv) nausea, (v) muscle pain or cramps, (vi) hypotension or hypertension episode and (vii) RPE $\geq 15$ according to Borg's scale.

\subsection{Anthropometric Profile}

Body mass was measured to the nearest $0.5 \mathrm{~kg}$ and body height to the nearest $0.5 \mathrm{~cm}$ by using a beam balance with stadiometer (Beam Balance-Stadiometer, SECA, Vogel \& Halke, Hamburg, Germany), as described previously [36]. BMI was calculated as the ratio of weight (kg) to height squared $\left(\mathrm{m}^{2}\right)$ and body fat percentage was determined by the measurement of 7 skinfolds using a Harpenden caliper (John Bull British Indicators Ltd., England) [36].

\subsection{Physical Performance}

Participants' $\mathrm{VO}_{2 \text { peak }}$ was determined during cardiopulmonary exercise stress testing on a stationary cycle ergometer (Monark 834E ergomedic testing bike, Varberg, Sweden) by using a pulmonary gas exchange system (Oxycon Mobile, Yorba Linda, CA, USA), as described [36]. Briefly, the workload was set at 10-20 W over the first $60 \mathrm{~s}$ and then was gradually increased by 5-10 W/min, until exhaustion. HR, 12-lead ECG, blood pressure and RPE were continuously monitored 
during the test and the subsequent recovery period, while a computerized system was utilized to assess oxygen uptake throughout the test via breath-by-breath analysis. The $\mathrm{VO}_{2}$ peak was determined as the maximum value on the $\mathrm{VO}_{2}$ curve where a plateau was occurred.

\subsection{Functional Capacity}

Participants' functional capacity was assessed using NSRI walk test and the sit-to-stand test (STS-60), as described [37,38]. Briefly, the NSRI walk test consisted of a 50-m indoor walk (flat ground), stair climbing (climbing up 22 stairs), stair descent (climbing down 22 stairs) and another $50 \mathrm{~m}$ walk to the start line. Participants received the instruction to execute the task as fast as possible, and were continuously motivated by the investigators. The total time (in seconds) taken to complete the task was recorded. The STS-60 test involves rising (knees fully extended) and sitting (contact with the chair) back on a chair (standard height) as fast as possible in $60 \mathrm{~s}$, without assistance and represents an indicator of muscle endurance. Participants were instructed to place their hands crossed over the chest and to keep their feet on the ground throughout the testing process. Furthermore, handgrip strength was also measured by using a Jamar hydraulic dynamometer, with participants sitting in standard chair as described [39].

\subsection{Quality of Life}

Self-reported quality of life was assessed using the SF-36 quality of life scoring system [38,40]. It involves 36 questions that are categorized into eight different scales: (i) physical functioning, (ii) role-physical, (iii) bodily pain, (iv) general health, (v) vitality, (vi) social functioning, (vii) role-emotional and (viii) mental health. These scales provide evaluation of two major dimensions: (i) physical health and (ii) mental health and self-evaluation. The average score of the eight scales was considered as the SF-36 final score.

\subsection{Blood Sampling and Assays}

Resting blood samples ( 12 mL) were collected pre- (1 day prior to the first training session) and post-training (5 days after the last training session). All samples were collected between 07:00-08:00 (to avoid circadian variations) after an overnight fast, from a forearm vein with subjects in a seated position. A blood portion $(\sim 6 \mathrm{~mL})$ was collected in Vacutainer tube, left to clot for 30 min and centrifuged $\left(1500 \times g, 15 \mathrm{~min}, 4^{\circ} \mathrm{C}\right)$ for serum separation. The supernatant (serum) was transferred to Eppendorf tubes and stored at $-80{ }^{\circ} \mathrm{C}$ for later analyses of TBARS, PC and hs-CRP. Another blood portion $(\sim 6 \mathrm{~mL})$ was collected into tubes containing ethylenediaminetetraacetic acid (EDTA), was immediately centrifuged $\left(1370 \times g, 4{ }^{\circ} \mathrm{C}, 10 \mathrm{~min}\right)$, and the plasma was collected and dispensed in multiple Eppendorf tubes and stored at $-80^{\circ} \mathrm{C}$ for later determination of TAC. The remaining plasma erythrocytes were lysed as described [41], and the lysate was later used for CAT, GSH and GSSG determination.

Hs-CRP concentration was quantitatively measured in serum with a latex-enhanced immunoturbidimetric assay, using the Roche Cobas clinical chemistry analyzer, as described [42]. TBARS, PC, TAC, CAT, GSH and GSSG were assayed according to protocols previously described [36,43-47]. Briefly, for the determination of TBARS $100 \mu \mathrm{L}$ of serum were mixed with $500 \mu \mathrm{L} 35 \%$ TCA and $500 \mu \mathrm{L}$ Tris-HCL (200 mM; pH 7.4) and incubated at room temperature for $10 \mathrm{~min}$. Then, $1 \mathrm{~mL}$ of $\mathrm{Na}_{2} \mathrm{SO}_{4}(2 \mathrm{M})$ - thiobarbituric acid (55 $\left.\mathrm{mM}\right)$ was added to the solution, and the solution was incubated at $95{ }^{\circ} \mathrm{C}$ for $45 \mathrm{~min}$. After incubation, samples were allowed to cool for $5 \mathrm{~min}$, mixed (vortex) after adding $1 \mathrm{~mL} \mathrm{70 \%} \mathrm{TCA,} \mathrm{centrifuged}$ $(15,000 \times g$, for $3 \mathrm{~min})$ and the absorbance of the supernatant was then measured at $530 \mathrm{~nm}$. PC were assayed by adding $50 \mu \mathrm{L}$ of serum to $50 \mu \mathrm{L}$ of $20 \%$ TCA prior to incubation in the ice bath for $15 \mathrm{~min}$ and immediately after incubation the solution was centrifuged $\left(15,000 \times g, 4{ }^{\circ} \mathrm{C}, 5 \mathrm{~min}\right)$. Then, the supernatant was discarded and $500 \mu \mathrm{L}$ of 2.4-dinitrophenylhydrazine (10 mM in $2.5 \mathrm{~N}$ HCL) for the samples or $500 \mu \mathrm{L}$ of HCL $(2.5 \mathrm{~N})$ for the blank was added to the remaining pellet. Thereafter, samples and blank solutions incubated in the dark at room temperature for $1 \mathrm{~h}$ with intermittent mixing every $15 \mathrm{~min}$. After incubation, samples and blank solutions centrifuged $\left(15,000 \times g, 4^{\circ} \mathrm{C}\right.$, 
$5 \mathrm{~min}), 1 \mathrm{~mL}$ of $10 \%$ TCA was added to the pellet after discarding the supernatant and centrifuged again at $15,000 \mathrm{~g}, 4{ }^{\circ} \mathrm{C}$ for $5 \mathrm{~min}$. The supernatant was then removed, $1 \mathrm{~mL}$ of ethanol-ethyl acetate $(1: 1 \mathrm{v} / \mathrm{v})$ was added to the pellet and centrifuged for $5 \mathrm{~min}\left(15,000 \times g, 4^{\circ} \mathrm{C}\right)$. The last procedure repeated two more times, and, thereafter, the supernatant was discarded, the pellet mixed with $1 \mathrm{~mL}$ of $5 \mathrm{M}$ urea ( $\mathrm{pH}$ 2.3) and incubated at $37^{\circ} \mathrm{C}$ for $15 \mathrm{~min}$. Finally, the samples and blank solutions centrifuged for $3 \mathrm{~min}\left(15,000 \times \mathrm{g}, 4^{\circ} \mathrm{C}\right)$ and the absorbance of the supernatant read at $375 \mathrm{~nm}$. For TAC analysis, $20 \mu \mathrm{L}$ of serum was mixed with $480 \mu \mathrm{L}$ of $10 \mathrm{mM}$ sodium-potassium phosphate ( $\mathrm{pH}$ 7.4) and $500 \mu \mathrm{L}$ of 2.2-diphenyl-1 picrylhydrazyl $(0.1 \mathrm{mM})$, incubated for $30 \mathrm{~min}$ in the dark, at room temperature, and centrifuged $(20,000 \times g$, for $3 \mathrm{~min})$ before having their absorbance read at $520 \mathrm{~nm}$. For the measurement of CAT activity, $4 \mu \mathrm{L}$ of red blood cell (RBC) lysate was added to $2991 \mu \mathrm{L}$ of $67 \mathrm{mM}$ sodium-potassium phosphate buffer ( $\mathrm{pH}$ 7.4), and the samples were mixed and incubated at $37^{\circ} \mathrm{C}$ for $10 \mathrm{~min}$. Subsequently, $5 \mu \mathrm{L}$ of $30 \%$ hydrogen peroxide was added in the samples and the change in absorbance was immediately read at $240 \mathrm{~nm}$ for $90 \mathrm{~s}$. For GSH determination, $20 \mu \mathrm{L}$ of RBC lysate was mixed with $660 \mu \mathrm{L}$ of $67 \mathrm{mM}$ sodium-potassium phosphate buffer ( $\mathrm{pH}$ 8.0) and $330 \mu \mathrm{L}$ of 5.5-dithiobis-2-nitrobenzoate $(1 \mathrm{mM})$, following treatment with $5 \% \mathrm{TCA}$, and then incubated for $45 \mathrm{~min}$ in the dark, at room temperature. Immediately after incubation, their absorbance was read at $412 \mathrm{~nm}$. For GSSG determination, RBC lysate was initially treated with 5\% TCA (pH 7.0-7.5). Following the addition of $4 \mu \mathrm{L}$ of 2-vinyl pyridine, samples were incubated at room temperature for $2 \mathrm{~h}$. After incubation, $600 \mu \mathrm{L}$ of sodium phosphate buffer (143 mM, pH 7.5), $100 \mu \mathrm{L}$ of NADPH ( $3 \mathrm{mM})$, $100 \mu \mathrm{L}$ of 5.5-dithiobis-2-nitrobenzoate $(10 \mathrm{mM})$ and $194 \mu \mathrm{L}$ of distilled water were added to samples and incubated at room temperature for $10 \mathrm{~min}$. Thereafter, $1 \mu \mathrm{L}$ of glutathione reductase was added and the change in absorbance was read at $412 \mathrm{~nm}$ for $3 \mathrm{~min}$.

\subsection{Statistical Analysis}

To ensure statistical power in our data analysis, we performed a power analysis prior to the study by using the GPower software (version 3.0.10) and setting an effect size $>0.55$, a probability error of 0.05 and a power of 0.90 for two groups and two measurement timepoints (pre- and post-training). The analysis indicated that a total sample size of 12 subjects is necessary to detect statistically meaningful differences among groups and timepoints. Accordingly, $24 \mathrm{HD}$ patients were included in the present study and 20 of them finally completed it and included in the analysis. Data are presented as means \pm standard deviation (SD). After verification of the normal distribution in our data sets (performed using the Shapiro-Wilk test), parametric tests were applied using the IBM SPSS software (IBM SPSS Statistics 20). A two-way (group $\times$ time) repeated measures analysis of variance (ANOVA) with planned contrasts on different time points was used to determine different time point changes on all dependent variables between the control and training groups. Effect sizes (ES) and confidence intervals (CI) were determined according to Hedge's g method corrected for bias. ES was interpreted as none, small, medium-sized, and large for values $0.00-0.19,0.20-0.49,0.50-0.79$, and $\geq 0.8$, respectively. Significance was accepted at $p<0.05$.

\section{Results}

The two groups were comparable at baseline (Table 1), and, as such, an analysis of covariance was not required. In CON 10 out of the 12 patients performed the follow up measurements (two dropouts). In the TR group, one patient underwent transplantation and was discontinued from the study, and one patient passed away. No musculoskeletal injuries or exercise-induced health-related complications were recorded. Consequently, 10 patients completed the study and the follow up assessments (a $81 \%$ compliance).

\subsection{External and Internal Load during the Training Intervention}

Participants in TR participated in a 6-month training intervention program incorporating a self-selected intensity and duration. The duration of exercise was significantly increased from $36 \%$ 
during the second month to $46 \%$ at the end of training. The external resistance during cycling was gradually increased from 0 to $60.6 \mathrm{~W}$ during the 6-month training period. Cycling frequency increased only in 6th month of training from 35 to 41.7 rounds/min. Despite this progressive increase in external load-related parameters, the internal load, i.e., mean systolic and diastolic blood pressure, mean HR, $\mathrm{RPE}$ and oxygen saturation remained unaltered throughout the training period. Table 2 presents the average monthly changes in external and internal load characteristics during the training intervention.

Table 2. Monthly changes in external and internal load during the 6-month intervention.

\begin{tabular}{ccccccc}
\hline Variables & 1st Month & 2nd Month & 3rd Month & 4th Month & 5th Month & 6th Month \\
\hline Duration of exercise (min) & $10.5 \pm 0$ & $16.5 \pm 1^{*}$ & $17 \pm 2.9^{*, \#}$ & $19.8 \pm 3.8^{*, \#}$ & $22 \pm 3.4^{*, \#}$ & $24 \pm 3.3^{*, \#}$ \\
Resistance (Watt) & $0 \pm 0$ & $20 \pm 14^{*}$ & $39.8 \pm 24^{*, \#}$ & $50.6 \pm 28^{*, \#}$ & $57 \pm 24^{*, \#}$ & $60.6 \pm 22^{*, \#}$ \\
Velocity (Rounds/min) & $35 \pm 0$ & $35 \pm 0$ & $35 \pm 0$ & $35 \pm 0$ & $35 \pm 0$ & $41.75 \pm 5.4^{*, \#}$ \\
Mean SBP (mmHg) & $148.64 \pm 13.8$ & $151.56 \pm 12.2$ & $152.62 \pm 13.1$ & $155.08 \pm 9.1$ & $152.16 \pm 11.8$ & $149.64 \pm 11.1$ \\
Mean DBP (mmHg) & $82.48 \pm 3.2$ & $81.06 \pm 7.6$ & $86.62 \pm 7.5$ & $84.98 \pm 4.2$ & $85.44 \pm 5.1$ & $81.52 \pm 7.1$ \\
Mean HR (beats/min) & $102.72 \pm 6.1$ & $104.78 \pm 6.9$ & $103.58 \pm 6.5$ & $102.5 \pm 6.1$ & $103.44 \pm 8.7$ & $103.32 \pm 8.7$ \\
RPE & $12.4 \pm 0.7$ & $12.1 \pm 0.6$ & $11.9 \pm 0.4$ & $12.6 \pm 0.7$ & $12.2 \pm 0.6$ & $11.8 \pm 0.3$ \\
SpO2 (\%) & $96.2 \pm 1.4$ & $97.3 \pm 1.3$ & $96.9 \pm 1.5$ & $96.4 \pm 1.4$ & $97.5 \pm 1.6$ & $96.6 \pm 1.5$ \\
\hline
\end{tabular}

SBP: Systolic blood pressure; DBP: Diastolic blood pressure; HR: Heart rate; RPE: Rate of perceived exertion. Data are presented as mean \pm standard deviation. * Significant difference with the 1st Month, \# Significant difference with the previous month.

\subsection{Somatometrics}

Body mass and body fat percentage decreased by almost $1 \mathrm{~kg}(p=0.013)$ and $2 \%(p=0.002)$, respectively, in TR, whereas, in CON, both variables remained unaltered (Table 3).

Table 3. Changes in participants' body composition.

\begin{tabular}{ccccc}
\hline Variables & Control Pre & Control Post & Experimental Pre & Experimental Post \\
\hline Body Height $(\mathrm{m})$ & \multicolumn{2}{c}{$1.71 \pm 0.1$} & \multicolumn{2}{c}{$1.71 \pm 0.09$} \\
Body Mass $(\mathrm{kg})$ & $74.6 \pm 9.3$ & $74.9 \pm 9.13$ & $72.5 \pm 14.6$ & $71.5 \pm 14^{*}$ \\
BMI $\left(\mathrm{kg} / \mathrm{m}^{2}\right)$ & $25.5 \pm 1.84$ & $25.6 \pm 3.19$ & $24.6 \pm 3.54$ & $24.2 \pm 3.39$ \\
Body Fat $(\%)$ & $27.3 \pm 3.54$ & $27.2 \pm 2.27$ & $27 \pm 2.27$ & $26.8 \pm 3.01 *$ \\
\hline
\end{tabular}

BMI: Body Mass Index. Data are presented as mean \pm standard deviation. * Significant difference with Pre.

\subsection{Physical Performance and Quality of Life}

Changes in physical performance and quality of life indicators are shown in Table 4 . Time to exhaustion $(+15 \% ; p<0.001) \mathrm{VO}_{2 \text { peak }}(+15 \% ; p<0.001)$ and blood lactate concentration $(+14 \% ; p<0.001)$ during the cardiopulmonary exercise stress test increased only in TR.

Similarly, performance was improved by $13 \%$ in STS-60 $(p=0.001)$, by $8 \%$ in NSRI $(p<0.001)$ and by $1 \%$ in handgrip strength testing $(p=0.002)$ only in TR post-training.

Subjective quality of life, as assessed through the SF-36 quality of life scoring system, increased post-training in TR by $4 \%(p=0.001)$ and was higher compared to CON $(p<0.001)$. In contrast, no significant changes were observed in SF-36 score in CON throughout the 6-month intervention period.

\subsection{Inflammation, Oxidative Stress and Antioxidant Status}

Figure 2 presents changes in inflammatory and OS markers in TR and CON. Following the 6-month training intervention, hs-CRP declined by $15 \%$ in TR $(p<0.001)$ and was lower compared to CON $(p<0.001)$ (Figure 2A). Likewise, TBARS declined by $28 \%(p<0.001)$ and PC by $31 \%(p<0.001)$ in TR post-training and were lower than CON (TBARS: $\mathrm{CON}=17.3 \pm 3.8 \mathrm{vs}$. $\mathrm{TR}=11.6 \pm 4.2 / \mathrm{PC}$ : $\mathrm{CON}=0.85 \pm 0.1$ vs. TR $=0.58 \pm 0.2 ; p<0.001$ ) (Figure $2 \mathrm{~B}, \mathrm{C}$ ). GSH increased by $52 \%$ post-training in TR $(p<0.001)$ and was higher compared to CON (CON: $1.12 \pm 0.44$ vs. TR: $1.72 \pm 0.45 ; p<0.001)$ (Figure 2D), while no changes were noted for GSSG (Figure 2E). The GSH/GSSG ratio increased by $51 \%$ in TR post-training $(p=0.001)$ and was higher than CON $(p=0.001)$ (Figure 2F). All OS indicators (i.e., hs-CRP, TBARS, PC, GSH, GSSG, GSH/GSSG) remained unaltered over time in CON (Figure 2A-F). 
Table 4. Changes in participants' physical performance status.

\begin{tabular}{ccccc}
\hline Variables & Control Pre & Control Post & Experimental Pre & Experimental Post \\
\hline VO $_{\text {2peak }}(\mathrm{ml} / \mathrm{kg} / \mathrm{min})$ & $14.8 \pm 3.1$ & $14.5 \pm 3.03$ & $13.81 \pm 3.03$ & $15.9 \pm 2.96^{*, \#}$ \\
Time to Exhaustion $(\mathrm{min})$ & $9.74 \pm 1.7$ & $9.64 \pm 1.69$ & $9.8 \pm 2.69$ & $11.3 \pm 2.16^{*, \#}$ \\
Resting HR (beats/min) & $79 \pm 9.9$ & $79.7 \pm 6.9$ & $78.4 \pm 13.2$ & $77 \pm 11.4$ \\
Peak HR (beats/min) & $122.9 \pm 17.6$ & $122.3 \pm 16.9$ & $121.5 \pm 17.3$ & $122.4 \pm 16.1$ \\
Resting Lactate $(\mathrm{mM})$ & $0.98 \pm 0.26$ & $1 \pm 0.19$ & $0.99 \pm 0.2$ & $0.97 \pm 0.17$ \\
Peak Lactate $(\mathrm{mM})$ & $6.1 \pm 1.1$ & $6.02 \pm 1.06$ & $6.2 \pm 1.6$ & $7.07 \pm 1.35^{*, \#}$ \\
STS-60 (reps in 60 s) & $33 \pm 7.6$ & $32.25 \pm 7$ & $33.83 \pm 7.2$ & $38.08 \pm 6.3^{*, \#}$ \\
NSRI test (s) & $51 \pm 8.78$ & $49.9 \pm 7.86$ & $49.8 \pm 10.6$ & $53.7 \pm 10.5^{*, \#}$ \\
Handgrip Strength (kg) & $24.7 \pm 9.65$ & $23 \pm 10.2$ & $23.4 \pm 10.5$ & $23.67 \pm 10.16^{*, \#}$ \\
SF-36 & $17.5 \pm 3.5$ & $17.6 \pm 2.6$ & $17.2 \pm 3.3$ & $15.3 \pm 3.1^{*, \#}$ \\
\hline
\end{tabular}

HR: Heart rate; STS-60: Sit-to-Stand - 60s test; NSRI: North Staffordshire Royal Infirmary; SF-36: Short form-36 quality of life scoring system. Data are presented as mean \pm standard deviation. * Significant difference with pre training intervention values; ${ }^{\#}$ Significant difference with the control group.
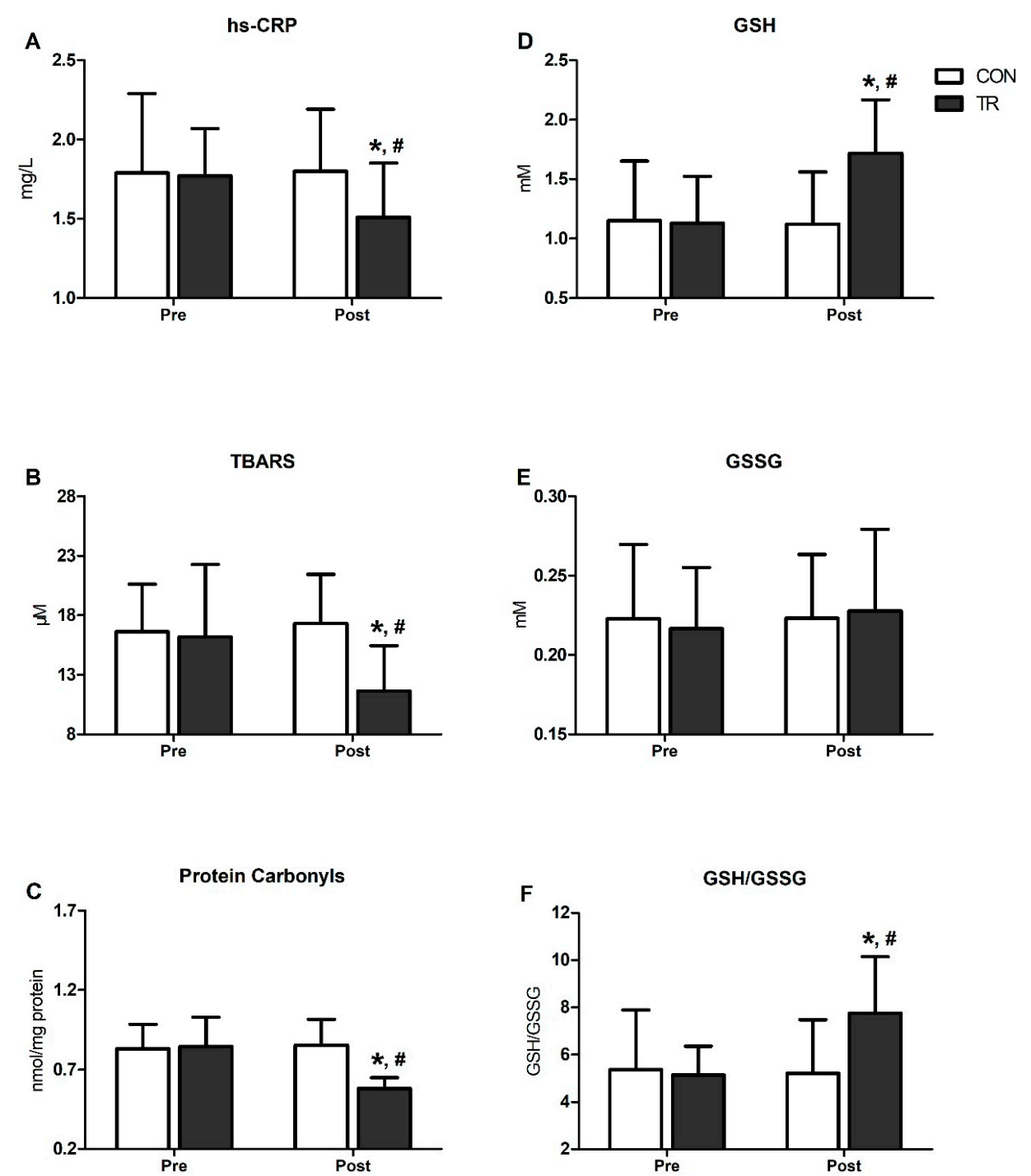

Figure 2. Six-month exercise training intervention reduced oxidative stress and systemic inflammation indices and improved blood redox status. Changes in high-sensitivity-CRP (hs-CRP) (A), thiobarbituric acid reactive substances (TBARS) (B), proteins carbonyls (C), reduced glutathione (GSH) (D), oxidized glutathione (GSSG) (E) and GSH/GSSG ration (F), in training group (TR) and control group (CON) after the 6-month intervention. Data are presented as mean \pm standard deviation. * Significant difference with pre-training values $(p<0.05)$; " Significant difference with the CON group $(p<0.05)$. 
TAC was substantially elevated post-training in TR by 59\% $(p<0.001)$ and was higher compared to CON ( $p<0.001)$ (Figure 3A). Similarly, CAT increased by $15 \%$ in TR post-training $(p<0.001)$ and was also higher than CON $(p<0.001)$ (Figure 3B). Both TAC and CAT remained unchanged in CON throughout the 6-month intervention period (Figure 3A,B).
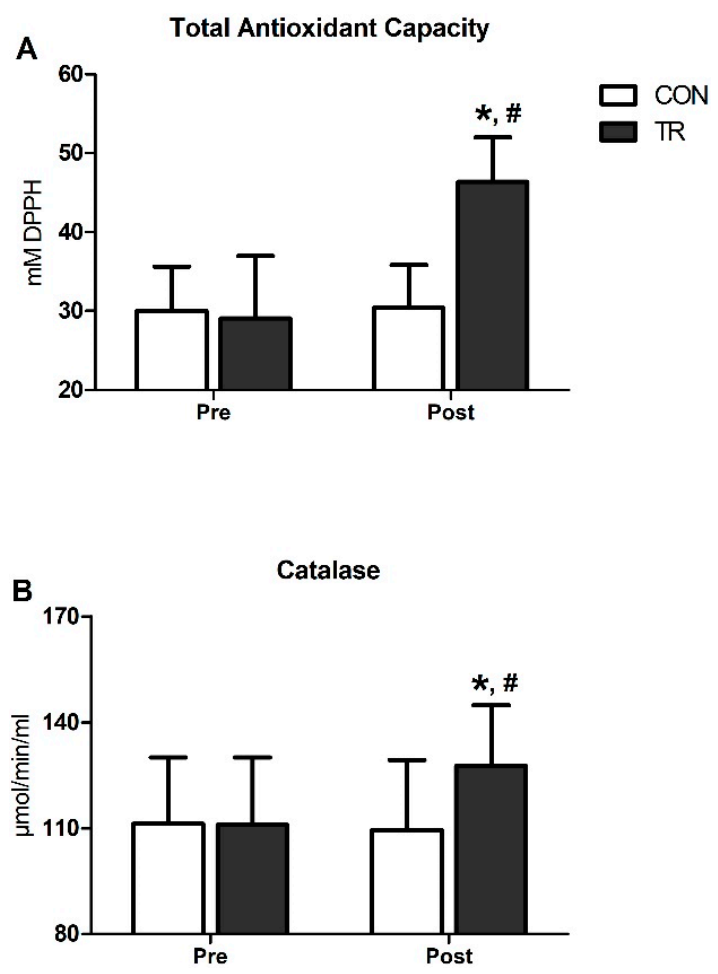

Figure 3. Six-month exercise training intervention upregulated total antioxidant capacity and catalase activity in blood. Changes in total antioxidant capacity (TAC) (A) and catalase (CAT) activity (B) in TR and $\mathrm{CON}$ after the 6-month intervention. Data are presented as mean \pm standard deviation. * Significant difference with pre-training values $(p<0.05)$; ${ }^{\#}$ Significant difference with the CON group $(p<0.05)$.

\section{Discussion}

Exercise training induced favorable changes in RS as well as in the physical and functional performance of patients on HD. Patients who participated in the 6-month exercise training program increased the external load during exercise, whereas internal load markers remained unchanged. Furthermore, TAC, CAT, GSH and GSH/GSSG were increased in patients who participated in the training process, while GSSG remained unaltered and TBARS and PC reduced. No dangerous adverse side effects were reported during exercise training. Only mild elevation of SBP and nausea were observed twice in different patients.

ESRD patients are suffering from muscle atrophy, reduced endurance and walking capacity which affect the quality of life and mortality [48]. Intradialytic exercise has been introduced as a non-pharmaceutical intervention to counteract the side effects of disease. Previous research papers have shown improvements in physical and functional performance [33,49-51]. STS-60 has been theorized as a sensitive functional marker to detect exercise-training improvements in ESRD patients [49,52]. Exercise training results in improvements of 5-14\% after 3 months and $~ 35 \%$ after 6 months [49,52]. These changes may reflect improvements in the quality of a patient's life as well. In agreement with previous studies, an improvement of 13\% in STS-60 was observed in this study in response to 6 months of training. In addition, we noticed a small, but significant increase in handgrip strength, despite the fact that we utilized a training intervention that was consisted of lower limb exercise (i.e., cycling). This beneficial effect might be associated with the observed improvement in overall quality of life, 
suggesting that participants in the TR group became more physically active during the 6-month training intervention (i.e., they were able to perform more physical activities during the day) and consequently the increase in daily activities promoted their handgrip strength.

Endurance capacity was assessed through $\mathrm{VO}_{2 \text { peak }}$, time to exhaustion $[33,50,51]$ and the NSRI test $[33,49,53]$. We observed a $15 \%, 13 \%$ and $8 \%$ improvement in $\mathrm{VO}_{2 \text { peak }}$, time to exhaustion and NSRI test, respectively. In the same line, Kouidi et al. [50] showed an improvement of $24.8 \%$ in $\mathrm{VO}_{2 \text { peak }}$ and $61.4 \%$ in time to exhaustion in the same patient population after a 1-year training. On the other hand, Groussard et al. [33] did not observed changes in $\mathrm{VO}_{2 \text { peak }}$ with training despite the fact that performance in the 6-min walk test improved by $11 \%$. NSRI has been reserved as functional performance marker. Sakkas et al. [51], showed a 29\% improvement in the NSRI test performance in end-stage kidney patients who participated in an intradialytic exercise training program further corroborating our findings. Differences among studies regarding results are probably related to different characteristics of the training protocol employed (e.g., duration of intervention program, duration of exercise and intensity). The duration of training intervention program seems to be a crucial factor, as longer training interventions have been associated with a more pronounced beneficial effect in a dose-dependent manner $[33,49,50,53]$. An additional factor that appears to influence the training-induced adaptations is the age of the patients since older patients exhibit more serious functional limitations such as musculoskeletal discomforts and fatigue during the $\mathrm{VO}_{2 \text { peak }}$ determination process $[33,54]$.

Following training, we observed a marked reduction in OS markers and enhancement of the antioxidant status indices in TR as compared to CON. Specifically, protein carbonylation and lipid peroxidation were substantially reduced, indicating lower oxidative damage to proteins and lipids, whereas levels of reduced glutathione (GSH) and the GSH/GSSG ratio were elevated, revealing an improvement in blood RS. Furthermore, the endogenous antioxidant defense mechanism was also up-regulated post-training, as evidenced by increased levels of TAC and CAT. Indeed, there is a plethora of studies showing that regular exercise training reduces oxidative damage and up-regulates the activity of antioxidant enzymes, increasing, as such, the resistance to ROS [25]. Beyond the apparent enhancement of TAC and CAT activity that clearly denotes increased antioxidant defense, the up-regulation of GSH observed post-training is of paramount importance. GSH is the most important thiol in cells that acts as a scavenger of free radicals and other reactive oxygen and nitrogen species via enzymatic reactions (mainly via the glutathione peroxidase reaction), mitigating the elevation of OS markers (i.e., PC, TBARS) [55]. It also contributes to the attenuation of GSH/GSSG reduction (redox status) under OS conditions, thus preserving redox status, and regulates redox-sensitive signal transduction pathways responsible for redox and cellular homeostasis [55]. There is a considerable body of evidence reporting that ESRD patients are characterized by extremely low GSH levels and a diminished GSH-scavenging system (i.e., lower GSH levels and reduced GSH peroxidase activity), which leads to exacerbations of OS markers $[10,11,17]$. Notably, the activity of GSH peroxidase is progressively attenuated as the disease progresses [56]; thus, it seems that OS and GSH deficiency interact in a vicious cycle during the disease, accelerating its progression. Consequently, although acute exercise induces a substantial reduction in blood GSH levels [36], chronic participation to exercise training amplifies GSH [34] and may, therefore, be considered as an efficient, non-pharmaceutical intervention to reduce OS and improve the antioxidant status in ESRD patients.

To the best of our knowledge, there is currently a limited amount of evidence regarding the RS responses to chronic exercise training in patients with ESRD [24]. In line with our findings, Wilund et al. [32] observed a significant reduction (by 38\%) in serum TBARS levels after 4 months of an endurance exercise training program (three intradialytic cycling sessions/week) in patients undergoing haemodialysis, and Groussard et al. [33] reported that F2 $\alpha$-isoprostanes were lower (by 36\%) after a 3-month intradialytic cycling exercise intervention in patients with CKD. Furthermore, by implementing low-intensity swimming exercise in the pool for 12 weeks, Pechter et al. [34] showed a significant reduction in lipid peroxidation and enhancement of reduced glutathione levels in patients with moderate renal failure. Therefore, similar to what is evident in healthy $[24,25]$ and other clinical 
populations [25], chronic participation to exercise training elicits beneficial redox changes in the context of ESRD, though acute exercise either exacerbates or has no impact on OS [30,35]. This exercise-mediated effect can be mechanistically explained by the hormesis theory, according to which regular exercise results in intermittent and transient ROS production and OS, stimulating redox-sensitive signaling pathways that promote protective adaptations against a higher future elevation in ROS levels and molecular damage [57].

In terms of systemic inflammation, serum hs-CRP was assessed as a surrogate marker of chronic inflammation in CKD $[58,59]$ and a strong predictor of atherogenic vascular risk and cardiovascular mortality in HD patients $[58,59]$. In contrast to a previous report where CRP levels remained unaltered in response to a 4-month intradialytic exercise training intervention (cycling) [32], we observed a 15\% reduction in hs-CRP in TR, in response to 6 months of exercise training. In fact, engaging in long-term cardiovascular exercise training, particularly of moderate intensity [60], is associated with a reduction in serum CRP levels both in healthy [61] and clinical populations [62,63], and is usually associated with reductions in body fat [61]. Accordingly, the reduction in hs-CRP levels observed in TR was accompanied by a $2 \%$ reduction in body fat percentage. In addition, our training program consisted of moderate-intensity bedside cycling (corresponding at a RPE of 11 ("light") to 13 ("somewhat hard")), suggesting that even this kind of moderate-intensity, non-weight-bearing exercise training is efficient in alleviating chronic inflammation in patients with ESRD.

However, one limitation in the current study is the fact that habitual physical activity was not monitored during the 6-month intervention period (i.e., neither on HD days nor on non-HD days). This would have enabled us to better understand the impact of our exercise training intervention per se on the dependent variables examined here, as the physical activity performed on HD and non-HD days might have induced and additive effect. Another possible limitation is that dietary macro- and micro-nutrient consumption was not recorded during the experimental period. Given that ESRD patients usually adhere to dietary restriction and/or malnutrition [9,11], a potential disparate dietary profile between our participants might have also interfered with the observed exercise training-induced adaptations.

\section{Conclusions}

Our study demonstrates that even low-to-moderate exercise intensity and duration of training may induce favorable improvements in functional and physical performance markers in ESRD patients. This investigation is the first to report a beneficial effect of low-to-moderate intensity cardiovascular exercise training on OS and systemic inflammation in these patients. Undoubtedly, future studies are required to examine this scenario using a larger sample size, to allow for the extraction of safe conclusions. In any case, ESRD patients should be encouraged to engage in cardiovascular exercise of low-to-moderate intensity as much as possible.

Author Contributions: All: writing of the manuscript. A.S., I.G.F., A.C., P.P., and V.V., conceptualized the study. S.P., E.N., performed participants medical screening. D.D., A.A., I.M., A.C., I.G.F., performed participants' functional and physical assessment. I.M., A.B., D.D., A.A., performed the training implementation. D.D., I.M., Y.M., performed assays related to RS and inflammation. All authors have read and agreed to the published version of the manuscript.

Funding: This research was supported by departmental (Department of Nephrology, Faculty of Medicine, University Hospital of Alexandroupolis, Democritus University of Thrace) funding only.

Conflicts of Interest: The authors declare no conflict of interest. 


\section{Abbreviations}

$\begin{array}{ll}\text { BMI } & \text { body mass index } \\ \text { CAT } & \text { catalase } \\ \text { CI } & \text { confidence intervals } \\ \text { CKD } & \text { chronic kidney disease } \\ \text { CON } & \text { control group } \\ \text { CVD } & \text { cardiovascular disease } \\ \text { DBP } & \text { diastolic blood pressure } \\ \text { DM } & \text { diabetes mellitus } \\ \text { EDTA } & \text { ethylene diamine tetraacetic acid } \\ \text { ES } & \text { effect sizes } \\ \text { ESRD } & \text { end-stage renal disease } \\ \text { GFR } & \text { glomerular filtration rate } \\ \text { GSH } & \text { redused glutathione } \\ \text { GSSG } & \text { oxidized glutathione } \\ \text { HD } & \text { hemodialysis } \\ \text { HR } & \text { heart rate } \\ \text { hs-CRP } & \text { high-sensitivity C-reactive protein } \\ \text { NSRI } & \text { north staffordshire royal infirmary } \\ \text { OS } & \text { oxidative stress } \\ \text { PC } & \text { protein carbonyls } \\ \text { PEW } & \text { protein wasting energy } \\ \text { ROS } & \text { reactive oxygen species } \\ \text { RPE } & \text { rate of perceived exertion } \\ \text { RRT } & \text { renal replacement therapy } \\ \text { RS } & \text { redox status } \\ \text { SaO } & \text { oxygen saturation } \\ \text { SBP } & \text { systolic blood pressure } \\ \text { SF-36 } & \text { short form-36 } \\ \text { STS-60 } & \text { sit to stand-60 } \\ \text { TAC } & \text { total antioxidant capacity } \\ \text { TBARS } & \text { thiobarbituric acid reactive substances } \\ \text { TCA } & \text { trichloro acetic acid } \\ \text { TR } & \text { training group } \\ \text { VO } & \text { peak oxygen consumption } \\ & \end{array}$

\section{References}

1. Jager, K.J.; Kovesdy, C.; Langham, R.; Rosenberg, M.; Jha, V.; Zoccali, C. A single number for advocacy and communication-Worldwide more than 850 million individuals have kidney diseases. Kidney Int. 2019, 96, 1048-1050. [CrossRef]

2. Jha, V.; Garcia-Garcia, G.; Iseki, K.; Li, Z.; Naicker, S.; Plattner, B.; Saran, R.; Wang, A.Y.-M.; Yang, C.-W. Chronic kidney disease: Global dimension and perspectives. Lancet 2013, 382, 260-272. [CrossRef]

3. Foreman, K.J.; Marquez, N.; Dolgert, A.; Fukutaki, K.; Fullman, N.; McGaughey, M.; A Pletcher, M.; E Smith, A.; Tang, K.; Yuan, C.-W.; et al. Forecasting life expectancy, years of life lost, and all-Cause and cause-Specific mortality for 250 causes of death: Reference and alternative scenarios for 2016-40 for 195 countries and territories. Lancet 2018, 392, 2052-2090. [CrossRef]

4. Chapter 1: Definition and classification of CKD. Kidney Int. Suppl. 2013, 3, 19-62. [CrossRef] [PubMed]

5. Saran, R.; Robinson, B.; Abbott, K.C.; Agodoa, L.Y.; Bhave, N.; Bragg-Gresham, J.; Balkrishnan, R.; Dietrich, X.; Eckard, A.; Eggers, P.W.; et al. US Renal Data System 2017 Annual Data Report: Epidemiology of Kidney Disease in the United States. Am. J. Kidney Dis. 2018, 71, A7. [CrossRef]

6. Duni, A.; Liakopoulos, V.; Roumeliotis, S.; Peschos, D.; Dounousi, E. Oxidative Stress in the Pathogenesis and Evolution of Chronic Kidney Disease: Untangling Ariadne's Thread. Int. J. Mol. Sci. 2019, 20, 3711. [CrossRef]

7. Zalba, G.; Fortuño, A.; Díez, J. Oxidative stress and atherosclerosis in early chronic kidney disease. Nephrol. Dial. Transplant. 2006, 21, 2686-2690. [CrossRef]

8. Dounousi, E.; Papavasiliou, E.; Makedou, A.; Ioannou, K.; Katopodis, K.P.; Tselepis, A.; Siamopoulos, K.C.; Tsakiris, D. Oxidative Stress Is Progressively Enhanced With Advancing Stages of CKD. Am. J. Kidney Dis. 2006, 48, 752-760. [CrossRef] 
9. Liakopoulos, V.; Roumeliotis, S.; Zarogiannis, S.G.; Eleftheriadis, T.; Mertens, P.R. Oxidative stress in hemodialysis: Causative mechanisms, clinical implications, and possible therapeutic interventions. Semin. Dial. 2018, 32, 58-71. [CrossRef]

10. Liakopoulos, V.; Roumeliotis, S.; Gorny, X.; Dounousi, E.; Mertens, P.R. Oxidative Stress in Hemodialysis Patients: A Review of the Literature. Oxidative Med. Cell. Longev. 2017, 2017, 1-22. [CrossRef]

11. Locatelli, F.; Canaud, B.; Eckardt, K.-U.; Stenvinkel, P.; Wanner, C.; Zoccali, C. Oxidative stress in end-stage renal disease: An emerging threat to patient outcome. Nephrol. Dial. Transplant. 2003, 18, 1272-1280. [CrossRef] [PubMed]

12. Podkowińska, A.; Formanowicz, D. Chronic Kidney Disease as Oxidative Stress- and Inflammatory-Mediated Cardiovascular Disease. Antioxidants 2020, 9, 752. [CrossRef] [PubMed]

13. Yang, C.-C.; Hsu, S.-P.; Wu, M.-S.; Chien, C.-T.; Hsu, S.-M. Effects of vitamin C infusion and vitamin E-coated membrane on hemodialysis-induced oxidative stress. Kidney Int. 2006, 69, 706-714. [CrossRef]

14. Poulianiti, K.P.; Kaltsatou, A.; Mitrou, G.I.; Jamurtas, A.Z.; Koutedakis, Y.; Maridaki, M.; Stefanidis, I.; Sakkas, G.K.; Karatzaferi, C. Systemic Redox Imbalance in Chronic Kidney Disease: A Systematic Review. Oxidative Med. Cell. Longev. 2016, 2016, 8598253. [CrossRef] [PubMed]

15. Tucker, P.S.; Scanlan, A.T.; Dalbo, V.J. Chronic Kidney Disease Influences Multiple Systems: Describing the Relationship between Oxidative Stress, Inflammation, Kidney Damage, and Concomitant Disease. Oxidative Med. Cell. Longev. 2015, 2015, 806358. [CrossRef] [PubMed]

16. Putri, A.Y.; Thaha, M. Role of oxidative stress on chronic kidney disease progression. Acta Med. Indones. 2014, 46, 244-252.

17. Daenen, K.; Andries, A.; Mekahli, D.; Van Schepdael, A.; Jouret, F.; Bammens, B. Oxidative stress in chronic kidney disease. Pediatr. Nephrol. 2018, 34, 975-991. [CrossRef]

18. Mihai, S.; Codrici, E.; Popescu, I.D.; Enciu, A.-M.; Albulescu, L.; Necula, L.G.; Mambet, C.; Anton, G.; Tanase, C. Inflammation-Related Mechanisms in Chronic Kidney Disease Prediction, Progression, and Outcome. J. Immunol. Res. 2018, 2018, 2180373. [CrossRef]

19. Genís, B.B.; Pastor, M.C.; Bonal, J.; Foraster, A.; Romero, R. Oxidative stress, inflammation and cardiovascular mortality in haemodialysis-Role of seniority and intravenous ferrotherapy: Analysis at 4 years of follow-up. Nephrol. Dial. Transplant. 2005, 21, 984-990. [CrossRef]

20. Fatouros, I.G.; Jamurtas, A.Z. Insights into the molecular etiology of exercise-induced inflammation: Opportunities for optimizing performance. J. Inflamm. Res. 2016, 9, 175-186. [CrossRef]

21. Michailidis, Y.; Karagounis, L.G.; Terzis, G.; Jamurtas, A.Z.; Spengos, K.; Tsoukas, D.; Chatzinikolaou, A.; Mandalidis, D.; Stefanetti, R.J.; Papassotiriou, I.; et al. Thiol-based antioxidant supplementation alters human skeletal muscle signaling and attenuates its inflammatory response and recovery after intense eccentric exercise. Am. J. Clin. Nutr. 2013, 98, 233-245. [CrossRef] [PubMed]

22. Fatouros, I.G.; Jamurtas, A.Z.; Villiotou, V.; Pouliopoulou, S.; Fotinakis, P.; Taxildaris, K.; Deliconstantinos, G. Oxidative Stress Responses in Older Men during Endurance Training and Detraining. Med. Sci. Sports Exerc. 2004, 36, 2065-2072. [CrossRef] [PubMed]

23. Nikolaidis, M.G.; Paschalis, V.; Giakas, G.; Fatouros, I.G.; Sakellariou, G.K.; Theodorou, A.A.; Koutedakis, Y.; Jamurtas, A.Z. Favorable and Prolonged Changes in Blood Lipid Profile after Muscle-Damaging Exercise. Med. Sci. Sports Exerc. 2008, 40, 1483-1489. [CrossRef] [PubMed]

24. Moinuddin, I.K.; Leehey, D.J. A Comparison of Aerobic Exercise and Resistance Training in Patients With and Without Chronic Kidney Disease. Adv. Chronic Kidney Dis. 2008, 15, 83-96. [CrossRef]

25. Sousa, C.V.; Sales, M.M.; Rosa, T.S.; Lewis, J.E.; De Andrade, R.V.; Simoes, H.G. The Antioxidant Effect of Exercise: A Systematic Review and Meta-Analysis. Sports Med. 2016, 47, 277-293. [CrossRef]

26. Smart, N.A.; McFarlane, J.; Cornelissen, V. The Effect of Exercise Therapy on Physical Function, Biochemistry and Dialysis Adequacy in Haemodialysis Patients: A Systematic Review and Meta-Analysis. Open J. Nephrol. 2013, 3, 25-36. [CrossRef]

27. Storer, T.W.; Casaburi, R.; Sawelson, S.; Kopple, J.D. Endurance exercise training during haemodialysis improves strength, power, fatigability and physical performance in maintenance haemodialysis patients. Nephrol. Dial. Transplant. 2005, 20, 1429-1437. [CrossRef]

28. Ouzouni, S.; Kouidi, E.; Sioulis, A.; Grekas, D.; Deligiannis, A. Effects of intradialytic exercise training on health-related quality of life indices in haemodialysis patients. Clin. Rehabil. 2009, 23, 53-63. [CrossRef] 
29. Chang, Y.; Cheng, S.-Y.; Lin, M.; Gau, F.-Y.; Chao, Y.-F.C. The effectiveness of intradialytic leg ergometry exercise for improving sedentary life style and fatigue among patients with chronic kidney disease: A randomized clinical trial. Int. J. Nurs. Stud. 2010, 47, 1383-1388. [CrossRef]

30. Böhm, J.; Monteiro, M.B.; Andrade, F.P.; Veronese, F.V.; Thomé, F.S. Acute effects of intradialytic aerobic exercise on solute removal, blood gases and oxidative stress in patients with chronic kidney disease. Braz. J. Nephrol. 2017, 39, 172-180. [CrossRef]

31. Levendoglu, F.; Altintepe, L.; Okudan, N.; Uğurlu, H.; Gökbel, H.; Tonbul, Z.; Guney, I.; Turk, S. A twelve week exercise program improves the psychological status, quality of life and work capacity in hemodialysis patients. J. Nephrol. 2004, 17, 826-832. [PubMed]

32. Wilund, K.R.; Tomayko, E.J.; Wu, P.-T.; Chung, H.R.; Vallurupalli, S.; Lakshminarayanan, B.; Fernhall, B. Intradialytic exercise training reduces oxidative stress and epicardial fat: A pilot study. Nephrol. Dial. Transplant. 2010, 25, 2695-2701. [CrossRef] [PubMed]

33. Groussard, C.; Rouchon-Isnard, M.; Coutard, C.; Romain, F.; Malardé, L.; Lemoine-Morel, S.; Martin, B.; Pereira, B.; Boisseau, N. Beneficial effects of an intradialytic cycling training program in patients with end-stage kidney disease. Appl. Physiol. Nutr. Metab. 2015, 40, 550-556. [CrossRef] [PubMed]

34. Pechter, U.; Ots, M.; Mesikepp, S.; Zilmer, K.; Kullissaar, T.; Vihalemm, T.; Zilmer, M.; Maaroos, J. Beneficial effects of water-based exercise in patients with chronic kidney disease. Int. J. Rehabil. Res. 2003, 26, 153-156. [CrossRef]

35. Esgalhado, M.; Stockler-Pinto, M.B.; Cardozo, L.F.M.D.F.; Costa, C.; Barboza, J.E.; Mafra, D. Effect of acute intradialytic strength physical exercise on oxidative stress and inflammatory responses in hemodialysis patients. Kidney Res. Clin. Pr. 2015, 34, 35-40. [CrossRef]

36. Fatouros, I.G.; Douroudos, I.; Panagoutsos, S.; Pasadakis, P.; Nikolaidis, M.G.; Chatzinikolaou, A.; Sovatzidis, A.; Michailidis, Y.; Jamurtas, A.Z.; Mandalidis, D.; et al. Effects of 1-Carnitine on Oxidative Stress Responses in Patients with Renal Disease. Med. Sci. Sports Exerc. 2010, 42, 1809-1818. [CrossRef]

37. Giannaki, C.D.; Stefanidis, I.; Karatzaferi, C.; Liakos, N.; Roka, V.; Ntente, I.; Sakkas, G.K. The Effect of Prolonged Intradialytic Exercise in Hemodialysis Efficiency Indices. ASAIO J. 2011, 57, 213-218. [CrossRef]

38. Koufaki, P.; Mercer, T. Assessment and Monitoring of Physical Function for People With CKD. Adv. Chronic Kidney Dis. 2009, 16, 410-419. [CrossRef]

39. Roberts, H.C.; Denison, H.; Martin, H.J.; Patel, H.P.; Syddall, H.; Cooper, C.; Sayer, A.A. A review of the measurement of grip strength in clinical and epidemiological studies: Towards a standardised approach. Age Ageing 2011, 40, 423-429. [CrossRef]

40. Kalantar-Zadeh, K.; Kopple, J.D.; Block, G.; Humphreys, M.H. Association among SF36 quality of life measures and nutrition, hospitalization, and mortality in hemodialysis. J. Am. Soc. Nephrol. 2001, 12, 2797-2806.

41. Theodorou, A.A.; Nikolaidis, M.G.; Paschalis, V.; Sakellariou, G.K.; Fatouros, I.G.; Koutedakis, Y.; Jamurtas, A.Z. Comparison between G6PD-Deficient and Normal Individuals after Eccentric Exercise. Med. Sci. Sports Exerc. 2009, 42, 1. [CrossRef] [PubMed]

42. Draganidis, D.; Jamurtas, A.Z.; Stampoulis, T.; Laschou, V.C.; Deli, C.K.; Georgakouli, K.; Papanikolaou, K.; Chatzinikolaou, A.; Michalopoulou, M.; Papadopoulos, C.; et al. Disparate Habitual Physical Activity and Dietary Intake Profiles of Elderly Men with Low and Elevated Systemic Inflammation. Nutrients 2018, 10, 566. [CrossRef] [PubMed]

43. Poulios, A.; Fatouros, I.G.; Mohr, M.; Draganidis, D.; Deli, C.K.; Papanikolaou, K.; Sovatzidis, A.; Nakopoulou, T.; Ermidis, G.; Tzatzakis, T.; et al. Post-Game High Protein Intake May Improve Recovery of Football-Specific Performance during a Congested Game Fixture: Results from the PRO-FOOTBALL Study. Nutrients 2018, 10, 494. [CrossRef] [PubMed]

44. Fatouros, I.G.; Pasadakis, P.; Sovatzidis, A.; Chatzinikolaou, A.; Panagoutsos, S.; Sivridis, D.; Michailidis, Y.; Douroudos, I.; Taxildaris, K.; Vargemezis, V. Acute Exercise May Exacerbate Oxidative Stress Response in Hemodialysis Patients. Nephron Clin. Pr. 2008, 109, c55-c64. [CrossRef]

45. Mohr, M.; Draganidis, D.; Chatzinikolaou, A.; Barbero-Alvarez, J.C.; Castagna, C.; Douroudos, I.; Avloniti, A.; Margeli, A.; Papassotiriou, I.; Flouris, A.D.; et al. Muscle damage, inflammatory, immune and performance responses to three football games in 1 week in competitive male players. Graefe's Arch. Clin. Exp. Ophthalmol. 2015, 116, 179-193. [CrossRef]

46. Chatzinikolaou, A.; Draganidis, D.; Avloniti, A.; Karipidis, A.; Jamurtas, A.Z.; Skevaki, C.L.; Tsoukas, D.; Sovatzidis, A.; Theodorou, A.; Kambas, A.; et al. The microcycle of inflammation and performance changes after a basketball match. J. Sports Sci. 2014, 32, 870-882. [CrossRef] 
47. Chatzinikolaou, A.; Christoforidis, C.; Avloniti, A.; Draganidis, D.; Jamurtas, A.Z.; Stampoulis, T.; Ermidis, G.; Sovatzidis, A.; Papassotiriou, I.; Kambas, A.; et al. A Microcycle of Inflammation Following a Team Handball Game. J. Strength Cond. Res. 2014, 28, 1981-1994. [CrossRef]

48. Roshanravan, B.; Gamboa, J.; Wilund, K. Exercise and CKD: Skeletal Muscle Dysfunction and Practical Application of Exercise to Prevent and Treat Physical Impairments in CKD. Am. J. Kidney Dis. 2017, 69, 837-852. [CrossRef]

49. Rhee, S.Y.; Song, J.K.; Hong, S.C.; Choi, J.W.; Jeon, H.J.; Shin, D.H.; Ji, E.H.; Choi, E.-H.; Lee, J.; Kim, A.; et al. Intradialytic exercise improves physical function and reduces intradialytic hypotension and depression in hemodialysis patients. Korean J. Intern. Med. 2019, 34, 588-598. [CrossRef]

50. Kouidi, E.; Karagiannis, V.; Grekas, D.; Iakovides, A.; Kaprinis, G.; Tourkantonis, A.; Deligiannis, A. Depression, heart rate variability, and exercise training in dialysis patients. Eur. J. Cardiovasc. Prev. Rehabil. 2010, 17, 160-167. [CrossRef]

51. Sakkas, G.K.; Hadjigeorgiou, G.M.; Karatzaferi, C.; Maridaki, M.; Giannaki, C.D.; Mertens, P.R.; Rountas, C.; Vlychou, M.; Liakopoulos, V.; Stefanidis, I. Intradialytic Aerobic Exercise Training Ameliorates Symptoms of Restless Legs Syndrome and Improves Functional Capacity in Patients on Hemodialysis. ASAIO J. 2008, 54, 185-190. [CrossRef] [PubMed]

52. Segura-Ortí, E.; Pérez-Domínguez, B.; De Villar, L.O.-P.; Oliva, E.M.; Gramage, J.M.; Maset, R.G.; Gil, J. Virtual reality exercise intradialysis to improve physical function: A feasibility randomized trial. Scand. J. Med. Sci. Sports 2018, 29, 89-94. [CrossRef] [PubMed]

53. Koh, K.P.; Fassett, R.G.; E Sharman, J.; Coombes, J.S.; Williams, A.D. Intradialytic versus home based exercise training in hemodialysis patients: A randomised controlled trial. BMC Nephrol. 2009, 10, 2. [CrossRef] [PubMed]

54. Matsuzawa, R.; Hoshi, K.; Yoneki, K.; Harada, M.; Watanabe, T.; Shimoda, T.; Yamamoto, S.; Matsunaga, A. Exercise Training in Elderly People Undergoing Hemodialysis: A Systematic Review and Meta-analysis. Kidney Int. Rep. 2017, 2, 1096-1110. [CrossRef]

55. Aquilano, K.; Baldelli, S.; Ciriolo, M.R. Glutathione: New roles in redox signaling for an old antioxidant. Front. Pharm. 2014, 5. [CrossRef] [PubMed]

56. Zachara, B.A.; Koterska, D.; Manitius, J.; Sadowski, L.; Dziedziczko, A.; Salak, A.; Wasowicz, W. Selenium Supplementation on Plasma Glutathione Peroxidase Activity in Patients with End-Stage Chronic Renal Failure. Biol. Trace Elem. Res. 2004, 97, 15-30. [CrossRef]

57. Radák, Z.; Chung, H.Y.; Goto, S. Exercise and hormesis: Oxidative stress-related adaptation for successful aging. Biogerontology 2005, 6, 71-75. [CrossRef]

58. Yeun, J.Y.; Levine, R.A.; Mantadilok, V.; Kaysen, G.A. C-reactive protein predicts all-cause and cardiovascular mortality in hemodialysis patients. Am. J. Kidney Dis. 2000, 35, 469-476. [CrossRef]

59. Zimmermann, J.; Herrlinger, S.; Pruy, A.; Metzger, T.; Wanner, C. Inflammation enhances cardiovascular risk and mortality in hemodialysis patients. Kidney Int. 1999, 55, 648-658. [CrossRef]

60. Fedewa, M.V.; Hathaway, E.D.; Higgins, S.; Forehand, R.L.; Schmidt, M.D.; Evans, E.M. Moderate, but not vigorous, intensity exercise training reduces C-reactive protein. Acta Cardiol. 2017, 73, 283-290. [CrossRef]

61. Fedewa, M.V.; Hathaway, E.D.; Ward-Ritacco, C.L. Effect of exercise training on C reactive protein: A systematic review and meta-analysis of randomised and non-randomised controlled trials. Br. J. Sports Med. 2016, 51, 670-676. [CrossRef] [PubMed]

62. Hammonds, T.L.; Gathright, E.C.; Goldstein, C.M.; Penn, M.S.; Hughes, J.W. Effects of exercise on c-reactive protein in healthy patients and in patients with heart disease: A meta-analysis. Hear. Lung 2016, 45, $273-282$. [CrossRef] [PubMed]

63. Saghebjoo, M.; Nezamdoost, Z.; Ahmadabadi, F.; Saffari, I.; Hamidi, A. The effect of 12 weeks of aerobic training on serum levels high sensitivity C-reactive protein, tumor necrosis factor-alpha, lipid profile and anthropometric characteristics in middle-age women patients with type 2 diabetes. Diabetes Metab. Syndr. Clin. Res. Rev. 2018, 12, 163-168. [CrossRef] [PubMed]

(C) 2020 by the authors. Licensee MDPI, Basel, Switzerland. This article is an open access article distributed under the terms and conditions of the Creative Commons Attribution (CC BY) license (http://creativecommons.org/licenses/by/4.0/). 